\title{
DERECHO INTERNACIONAL Y CONSTITUCIONAL SOBRE CONSULTA PREVIA EN PROYECTOS VIALES EN COLOMBIA
}

\section{Jairo Vladimir Llano Franco}

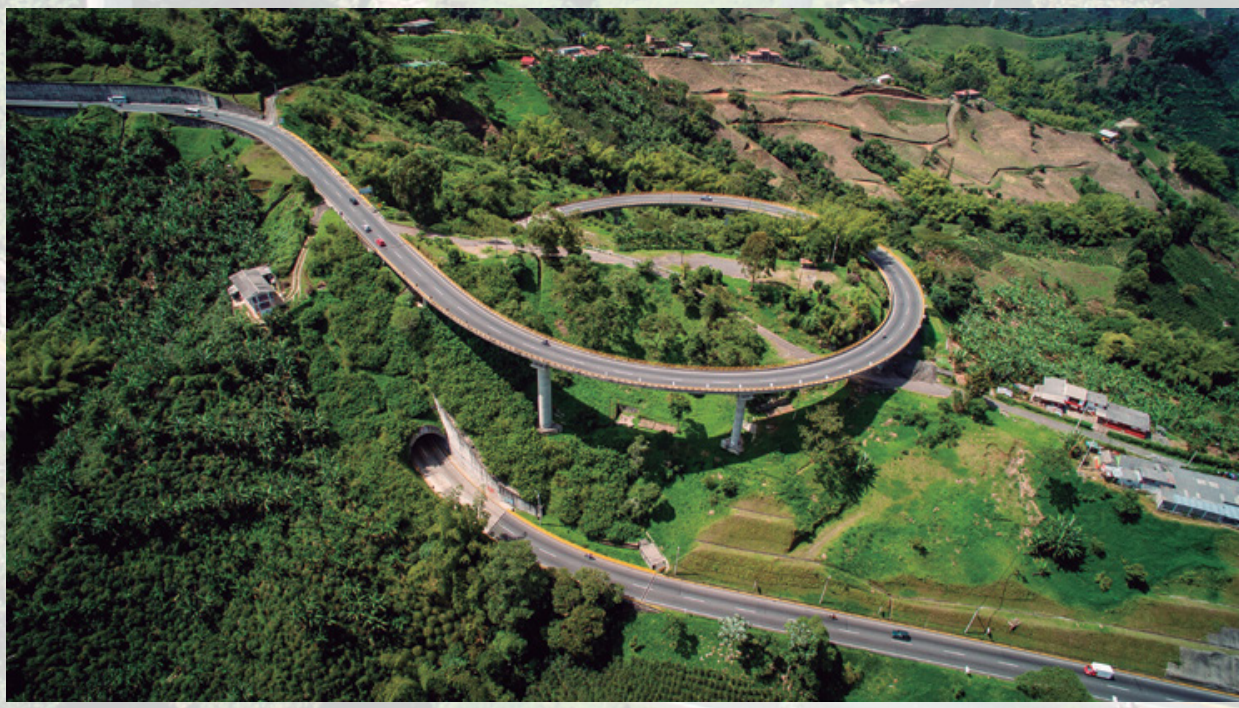





\title{
DERECHO INTERNACIONAL Y CONSTITUCIONAL SOBRE CONSULTA PREVIA EN PROYECTOS VIALES EN COLOMBIA
}

\author{
Jairo Vladimir Llano Franco \\ Universidad Católica de Colombia
}

\section{Resumen}

El artículo expondrá el origen del derecho fundamental de la consulta previa en el derecho internacional y su posterior vinculación por medio de la ratificación de tratados internacionales de derechos humanos que reconocen la diversidad cultural global y el bloque de constitucionalidad y la jurisprudencia de la Corte Constitucional. Con este soporte sociojurídico se ha pretendido garantizarlo en situaciones específicas como la concreción de proyectos de intervención en comunidades, entre los cuales se encuentra la transformación e innovación de infraestructura vial que se ha intensificado en los años recientes. Un caso es la doble calzada en la vía Panamericana en el trayecto Popayán-Santander de Quilichao en el departamento del Cauca, donde la diversidad étnica es predominante, el cual será analizado para determinar cómo se garantiza el derecho fundamental de la consulta previa en estos ambiciosos proyectos.

Palabras clave: consulta previa, derechos fundamentales, comunidades étnicas, indígenas, proyectos viales.

El autor: doctor en Derecho, antropólogo, especialista en Antropología Jurídica. Profesor e investigador del grupo Phronesis de la Facultad de Derecho de la Universidad Católica de Colombia. ORCID: 0000-0002-4018-5412. Correo electrónico: jairoderecho1@hotmail.com

Recibido: 14 de octubre de 2021; evaluado: 25 de noviembre de 2021; aceptado: 30 de noviembre de 2021. 


\title{
INTERNATIONAL AND CONSTITUTIONAL LAW ON PRIOR CONSULTATION IN ROAD PROJECTS IN COLOMBIA
}

\author{
Jairo Vladimir Llano Franco \\ Universidad Católica de Colombia
}

\begin{abstract}
This article discusses the origin of the fundamental right to prior consultation in international law and its subsequent linkage through the ratification of international human rights treaties that recognize cultural diversity at a global level and the constitutionality block through the jurisprudence of the Constitutional Court. This socio-legal support is intended to guarantee this fundamental right in specific situations, such as the implementation of intervention projects in ethnic communities, including the transformation and innovation of road infrastructure that has intensified in recent years. One case is the double roadway on the Pan-American Highway on the Popayán-Santander de Quilichao route in the department of Cauca, where ethnic diversity is predominant, which is analyzed to determine how the fundamental right of prior consultation is guaranteed in ambitious projects of road infrastructure in Colombia.
\end{abstract}

Keywords: prior consultation, fundamental rights, ethnic, indigenous communities, road projects.

The author: $\mathrm{PhD}$ in Law, BA in Anthropology, Specialization in Legal Anthropology. Professor and researcher of the Phronesis group of the Law School at the Universidad Católica de Colombia. ORCID: 0000-0002-4018-5412. E-mail: jairoderechol@hotmail.com

Received: October 14, 2021; evaluated: November 25, 2021; accepted: November 30, 2021. 


\title{
DIREITO INTERNACIONAL E CONSTITUCIONAL SOBRE A CONSULTA PRÉVIA EM PROJETOS VIÁRIOS NA COLÔMBIA
}

\author{
Jairo Vladimir Llano Franco \\ Universidad Católica de Colombia
}

\section{Resumo}

Este artigo expõe a origem do direito fundamental da consulta prévia no Direito Internacional e sua posterior vinculação por meio da ratificação de tratados internacionais de Direitos Humanos que reconhecem a diversidade cultural global e o conjunto de constitucionalidade, bem como da jurisprudência da Corte Constitucional. Com esse suporte sociojurídico, pretendeu-se garanti-lo em situações específicas como a concretização de projetos de intervenção em comunidades, entre os quais se encontram a transformação e inovação de infraestrutura viária que vêm se intensificando nos anos recentes. Um caso é a pista dupla na rodovia Panamericana no trajeto Popayán-Santander de Quilichao no estado do Cauca, Colômbia, onde a diversidade étnica é predominante, o que é analisado para determinar como o direito fundamental à consulta prévia é garantido nesses ambiciosos projetos.

Palavras-chave: consulta prévia, direitos fundamentais, comunidades étnicas, indígenas, projetos viários.

O autor: doutor em Direito. Antropólogo. Especialista em Antropologia Jurídica. Professor e pesquisador do grupo Phronesis da Faculdade de Direito da Universidad Católica de Colombia. ORCID: 0000-0002-4018-5412. Correio eletrônico: jairoderecho1@hotmail.com

Recebido: 14 de outubro de 2021; avaliado: 25 de novembro de 2021; aceito: 30 de novembro de 2021. 


\section{Introducción}

El derecho a la consulta previa para adelantar proyectos de intervención en territorios de ciertas comunidades en la década de 1990 se percibía como una propuesta innovadora, que se encontraba en concordancia con el reconocimiento de la diversidad étnica y cultural plasmado formalmente en la Constitución colombiana. Incluso, las instituciones estatales y las empresas privadas lo consideraban una acción plausible para llevar a cabo sus proyectos en regiones de difícil acceso y con las comunidades que tienen cosmovisiones diferentes al resto del país; sin embargo, con el tiempo y la implementación de este derecho fundamental, la percepción se ha venido transformando.

Al implementar los procesos de consulta previa, las entidades públicas y privadas observaron que los costos se incrementaban, pues la inversión se ampliaba a la realización de la consulta, debido a los compromisos adquiridos con la comunidad y a las compensaciones por los daños ocasionados por la intervención. Estos sobrecostos eran asumidos por los encargados de llevar a cabo el proyecto, situación que originó de manera reciente propuestas de funcionarios y empresarios de reformar los procesos de consulta para beneficio de los ejecutores. Esto ha impulsado, con sutileza, el desmonte de este derecho fundamental.

Para comprender estas tensiones y revisar las implicaciones que conlleva garantizar este derecho en el contexto colombiano, en la primera parte del artículo se expone el origen de la consulta previa en el derecho internacional. En la segunda, se muestra su consolidación por la vinculación a las declaraciones internacionales recientes. En la tercera sección se indica que la Corte Constitucional ha sido esencial, no solo para el reconocimiento del derecho fundamental, sino también para su implementación. En la cuarta y la quinta sección se aborda la consulta previa en proyectos viales mediante un estudio de caso: el proyecto de doble calzada en la vía Panamericana en el trayecto Popayán-Santander de Quilichao, que afectará a las comunidades indígenas nasa.

Este es un trabajo de sociología jurídica, adscrito a la tradición de pensamiento que, en América Latina, se ha preocupado por constatar la eficacia de las disposiciones legales en las prácticas sociales. ${ }^{1}$ También representa la perspectiva del nuevo

Germán Silva García et al., "El desarrollo de la sociología jurídica latinoamericana", Opción 35, núm. 25 esp. (2019): 1136-1196; Jorge Enrique Carvajal-Martínez, La sociología jurídica en Colombia (Bogotá: Universidad Libre, 2016); Germán 
derecho constitucional, que toma como eje los derechos fundamentales y tiene como propósito examinar el papel del derecho en la protección de los grupos sociales más vulnerables. ${ }^{2}$

\begin{abstract}
Asimismo, da continuidad a los trabajos que han ahondado en la cuestión de los derechos humanos, ${ }^{3}$ profundamente conectada con el derecho a la consulta previa de las comunidades, que se plantea en un país con un grave historial de transgresiones. ${ }^{4}$ Esa línea de desarrollo está ligada a un conjunto de investigaciones que, de modo crítico, exploran respuestas sociojurídicas apropiadas para el Sur Global, ajustadas a sus características singulares y no a los moldes teóricos del Norte. ${ }^{5}$
\end{abstract}

Silva García, "Historia de la sociología jurídica latinoamericana. Los que se fueron... y su herencia. Manuel Calvo García", Revista Latinoamericana de Sociología Jurídica 2, núm. 3, (2021): 5-21.

2 Jairo Vladimir Llano Franco y Germán Silva García, "Globalización del derecho constitucional y constitucionalismo crítico en América Latina", Utopía y Praxis Latinoamericana 23, núm. 2 extra (2018): 59-

73; Germán Silva García, "La administración de justicia: ¿escenario para la protección de los grupos sociales vulnerables?", Revista Colombiana de Sociología, núm. 26 (2006): 105-123.

3 José-Manuel Barreto, "Decolonial Thinking and the Quest for Decolonising Human Rights", Asian Journal of Social Science 46, núm. 4-5 (2018): 484-502; Jaime Alfonso Cubides-Cárdenas, Paola Alexandra Sierra-Zamora, Diego Alexander Calixto Ortiz y Nicolás Pabón-Caballero, "Terrorismo por parte de las FARC-EP y políticas públicas orientadas a la seguridad nacional en Colombia durante 1990-2000", Revista Científica General José María Córdova 17, núm. 26 (2019): 308-325; Camilo Alberto Moncayo Zamudio, "Derechos humanos: ¿de la universalidad a la particularidad?", Nuevos Paradigmas de las Ciencias Sociales Latinoamericanas 10, núm. 19 (enero-junio 2019): 23-38; Bernardo Pérez-Salazar, "Construcción de paz en el orden del derecho transnacional penal: el caso colombiano", Utopía y Praxis Latinoamericana 23, núm. 1 (2018): 65-78; Germán Silva García, "Corrupción y derechos humanos. El Estado hacendal y la cleptocracia", Opción 35, núm. 25 esp. (2019): 12-49.

$4 \quad$ Tania Giovanna Vivas-Barrera y Bernardo Pérez Salazar, "Sobre la situación de graves violaciones de los derechos humanos y el derecho internacional humanitario en contra de las mujeres en el conflicto armado colombiano" en Derechos humanos, paz y posconflicto en Colombia, ed. Tania Giovanna Vivas-Barrera (Bogotá: Universidad Católica de Colombia, 2016), 59-104; María Elizabeth Valero Rico, "Reconstrucción del tejido social en Colombia a partir de la niñez víctima del conflicto", Nuevos Paradigmas de las Ciencias Sociales Latinoamericanas 7, núm. 13 (enero-junio 2016): 189-220; Aracelis Isabel Camacho Bustamante, "Las leyes de justicia y paz, de reparación y restitución de tierras y sus efectos en la sociedad colombiana", Nuevos Paradigmas de las Ciencias Sociales Latinoamericanas 10, núm. 20 (2019): 85-94; Rafael Velandia Montes, "Proceso de paz en Colombia y justicia penal internacional", Revista Latinoamericana de Sociología Jurídica 1, núm. 1 (2020): 346-371; Mayerling López Echeverri, "Privación de la libertad y resocialización en Colombia: ipopulismo punitivo?", Nuevos Paradigmas de las Ciencias Sociales Latinoamericanas 11, núm. 21 (2020): 21-33; Luis Felipe Dávila y Caroline Doyle, "Insider and outsider fieldwork challenges in Medellín, Colombia", International Journal for Crime, Justice and Social Democracy 9, núm. 3 (2020): 87-99; Pablo Elías González Monguí, "La selectividad penal negativa de las muertes ilegítimamente presentadas como bajas en combate por la fuerza pública del Estado colombiano", Revista Latinoamericana de Sociología Jurídica 1, núm. 1. (2020): 305-345; Jorge Naranjo Álvarez, "La violencia sexual a la luz de la ley de justicia y paz: conceptualización y elementos para su estudio", Novum Jus 15, núm. 2 (2021): 91-119.

5 Salo de Carvalho, "Criminología, garantismo y teoría crítica de los derechos humanos: ensayo sobre el ejercicio de los poderes punitivos", Novum Jus 3, núm. 1 (2009): 161-200; José-Manuel Barreto, "Decolonial strategies and dialogue in the human rights field: A manifesto", Transnational Legal Theory 3, núm. 1 (2012): 1-29; Nicole Velasco Cano y Jairo Vladimir Llano Franco, "Derechos fundamentales: un debate desde la argumentación jurídica el garantismo y el comunitarismo", Novum Jus 10, núm. 2 (2016): 35-55; Germán 


\section{Orígenes de la consulta previa desde el derecho internacional}

Los primeros avances en el reconocimiento de la diversidad étnica y cultural se han hecho desde el derecho internacional, con efectos en los Estados-nación. Una de las dificultades que ha caracterizado a este tipo de organización social y política de la Modernidad ha sido el desconocimiento o la invisibilidad de las comunidades o los pueblos considerados diferentes respecto a la sociedad nacional, debido a que la pretensión del Estado moderno es la integración de los ciudadanos con una identidad específica: "[El] Estado necesita de la fidelidad y la dedicación de sus ciudadanos: la nación es el nuevo y atrayente símbolo de identidad y de pertenencia [...] en una herramienta eficaz de integración y de sujeción de los individuos". ${ }^{6}$

Ante esta configuración de los Estados-nación, en el contexto latinoamericano, la diferencia cultural fue ocultada o marginada en el mejor de los casos. Por esta situación se acude a los tratados, convenios y pactos internacionales, caracterizados por la inclusión de lo diferente. Estos documentos han logrado ampliar su incidencia en lo estatal, pues pasaron de un discurso general sin mayores efectos jurídicos y administrativos a convertirse en vinculantes para los Estados. Dicha transformación tiene en las organizaciones supraestatales y las ONG de derechos humanos transnacionales los promotores de los derechos culturales y de la diversidad en el ámbito internacional: "Fue solamente después de haberse planteado el problema de las poblaciones indígenas en la Comisión de Derechos Humanos de la ONU, que el Consejo Económico y Social aprobó [... ] un estudio sobre la discriminación contra las poblaciones indígenas". ${ }^{7}$

Es sorprendente que, pese a los avances académicos e investigativos de las disciplinas de las ciencias sociales como la antropología, la sociología y la ciencia política respecto a la diversidad cultural, no sean recogidos por las instituciones estatales responsables para la realización de sus acciones que pretenden la garantía

Silva García y Bernardo Pérez-Salazar, "Nuevas estrategias de construcción de la realidad del delito en el orden de las sociedades en red", Utopía y Praxis Latinoamericana 24, núm. 2 extra (2019): 123-132; Pablo Elías González Monguí, "La negación de la calidad de ciudadano o de persona en el derecho penal de enemigo", Opción 35, núm. 25 esp. (2019): 1070-1103; Germán Silva García, Fabiana Irala y Bernardo Pérez-Salazar, "Criminalidad, desviación y divergencia. Una nueva cosmovisión en la criminología del sur", Revista Latinoamericana de Sociología Jurídica 1, núm. 1 (2020): 8-32.

6 Pietro Costa, "Ciudadanía y patrones de pertenencia a la comunidad política" en Nacionalidad y ciudadanía, eds. Pietro Costa y Benito Aláez Corral (Madrid: Fundación Coloquio Jurídico Europeo, 2010), 30.

7 Rodolfo Stavenhagen, Derecho indígena y derechos humanos en América Latina (Ciudad de México: Instituto Interamericano de Derechos Humanos, 2012), 349. 
y protección de esta diversidad; en repetidas ocasiones, por desconocimiento, originan lo contrario: exclusión y marginalidad de las poblaciones diferenciadas que se pretendían reconocer.

En repetidas ocasiones, las reivindicaciones de los pueblos indígenas latinoamericanos tienen mayor reconocimiento en la esfera internacional del Norte Global que en su propia región, donde es común asistir a la configuración de sectores sociales que rechazan a sus pueblos originarios y sus movilizaciones reivindicativas. Esta acogida internacional facilita que sus procesos de consolidación de identidad cultural continúe y no sean truncados por los intereses de las empresas que pretenden explotar sus recursos naturales: "El relato étnico identitario promovió reivindicaciones y reclamos en escenarios internacionales [...] sobre temas asociados con la desigualdad, y ganaron apoyos y alianzas por medio de la autopista global [...] identidad étnica global determinante para alcanzar logros jurídico-políticos internacionales". ${ }^{8}$

El derecho a la consulta previa tiene sus orígenes en el derecho internacional, en relación con el reconocimiento de los pueblos indígenas y las comunidades consideradas diferenciadas. Un texto sociojurídico internacional de transcendencia que llevó a plasmar la consulta es el Convenio 169 promulgado por la supraestatal Organización Internacional del Trabajo en 1989 sobre pueblos indígenas y tribales, con un impulso a la autonomía de las comunidades indígenas: "[... ] la reivindicación de los derechos de los pueblos indígenas opuesto al constitucionalismo liberal [...] descansa en una visión pluralista, que intenta dejar atrás tanto las políticas asimilacionistas como integracionistas".

Este Convenio ha tenido el respaldo de un número significativo de países latinoamericanos que lo han ratificado, por lo que se convirtió en un instrumento sociojurídico internacional que ha sido apropiado por los Gobiernos y los mismos pueblos diferenciados de la región. Así, "la OIT adoptó el Convenio 169, que ha sido ratificado por 22 países, 14 de los cuales pertenecen a América Latina (Argentina,

8 Nasly Nayely Cruz Chavarro y Gabriel Andrés Arévalo Robles, "Cumbres indígenas: política y diplomacia ancestral en América Latina", Novum Jus 15, núm. 1(2021): 142, DOI: https://doi.org/10.14718/ NovumJus.2021.15.1.7 (acceso noviembre 10, 2021).

9 María Dolores Núñez Ávila, "El reconocimiento de 'la justicia indígena' como agente de la reivindicación de los pueblos indígenas en el marco del pluralismo jurídico", Anuario Iberoamericano de Derecho Internacional Penal 6, núm. 6 (2018): 182, DOI: https://doi.org/10.12804/revistas.urosario.edu.co/anidip/a.7162 (acceso octubre 15, 2021). 
Bolivia, Brasil, Chile, Colombia, Costa Rica, Ecuador, Guatemala, Honduras, México, Nicaragua, Paraguay, Perú y Venezuela)". ${ }^{10}$

Entre los artículos que impulsan el derecho fundamental de la consulta previa para los pueblos indígenas y tribales de forma precisa se encuentran: "[...] los gobiernos deberán a) consultar a los pueblos interesados mediante procedimientos apropiados y en particular a través de sus instituciones representativas, cada vez que se prevean medidas legislativas o administrativas susceptibles de afectarles directamente". ${ }^{11}$

Aquí se dispone que la consulta previa se llevará a cabo ante medidas y decisiones gubernamentales que afecten a las comunidades indígenas y tribales que se encuentran en sus territorios.

El Convenio expone la consulta previa como la acción que posibilita acuerdos con los pueblos indígenas en el momento en que una decisión les concierna. Lograr que los indígenas permitan una determinada intervención en su territorio concretaría el consentimiento, que es esencial para adelantar un proyecto sin dificultades. "Las consultas llevadas a cabo en aplicación de este Convenio deberán efectuarse de buena fe y de una manera apropiada a las circunstancias, con la finalidad de llegar a un acuerdo o lograr el consentimiento acerca de las medidas propuestas". ${ }^{12}$ Más adelante, se solicita a los Gobiernos la vinculación de los pueblos indígenas y tribales a las acciones correspondientes al desarrollo socioeconómico del país en donde se lleven a cabo intervenciones estatales que afecten sus territorios colectivos y su identidad cultural: "Los pueblos interesados deberán tener derecho a decidir sus propias prioridades en lo que atañe al proceso de desarrollo, en la medida que éste afecte sus vidas, creencias, instituciones y bienestar espiritual y a las tierras que ocupan". ${ }^{13}$ En cuanto al mejoramiento de las condiciones de vida por parte de las instituciones estatales mediante los planes de desarrollo hacia los pueblos diferenciados, también debe consultarse:

[...] mejoramiento de las condiciones de vida y de trabajo y del nivel de salud y educación de los pueblos interesados, con su participación y cooperación,

10 Organización Internacional del Trabajo [OIT], "El Convenio 169 de la OIT". https://www.ilo.org/ global/docs/WCMS_236378/lang--es/index.htm\#: :text=En\%201989\%2C\%20despu\%C3\%A9s\%20de\%20 dos,Paraguay\%2C\%20Per\%C3\%BA\%20y\%20Venezuela (acceso 20 de mayo, 2021).

11 Oficina Internacional del Trabajo [OIT], Convenio Núm. 169 de la OIT sobre pueblos indígenas y tribales (Ginebra, 27 de junio de 1989), art. 6, num. 1, lit. a.

12 OIT, Convenio Núm. 169, art. 6, num. 2.

13 OIT, Convenio Núm. 169, art. 7, num. 1. 
deberá ser prioritario en los planes de desarrollo económico global de las regiones donde habitan. ${ }^{14}$

\section{La consulta previa en el derecho internacional del siglo XXI}

Al Convenio 169 de la OIT, que reconoce la consulta previa como un derecho fundamental de carácter internacional, se le vinculan otros textos jurídicos que posicionan de manera global la implementación de este derecho fundamental que beneficia a las comunidades, con un especial interés en los pueblos indígenas. Es el caso de la Declaración sobre los derechos de los pueblos indígenas de 2007: "Los Estados celebrarán consultas y cooperarán de buena fe con los pueblos indígenas interesados por conducto de sus propias instituciones representativas a fin de obtener su consentimiento libre e informado antes de aprobar cualquier proyecto que afecte sus tierras". ${ }^{15}$

En seguida, la Declaración expone la reparación que deben hacer los Estados y los particulares a las comunidades cuando implementan proyectos de intervención en los territorios que pertenecen a los pueblos indígenas: "Los Estados proveerán mecanismos eficaces para la reparación justa y equitativa por cualquiera de esas actividades, y se adoptarán medidas adecuadas para mitigar las consecuencias nocivas de orden ambiental, económico, social, cultural o espiritual". ${ }^{16}$

Un artículo interesante de la Declaración se relaciona con las acciones y los operativos de las fuerzas militares en territorios indígenas. Para llevar a cabo estas intervenciones militares, se requiere la consulta previa: "Artículo 30.1. No se desarrollarán actividades militares en las tierras o territorios de los pueblos indígenas, a menos que se justifique una razón de interés público pertinente o que se haya acordado libremente con los pueblos indígenas". ${ }^{17}$ Para los países con conflicto armado, como el colombiano, el respaldo de este artículo de la Declaración se convierte en prioritario para la protección de los pueblos; sin embargo, los operativos, la instalación de bases y la presencia de tropas militares en sus territorios se ha vuelto constante. Por el contrario, en los territorios donde están ubicadas las comunidades indígenas,

OIT, Convenio Núm. 169, art. 7, num. 2.

15 ONU, Declaración de las Naciones Unidas sobre los derechos de los pueblos indígenas (Nueva York, 13 de septiembre de 2007), art. 32, num. 2.

16 ONU, Declaración de las Naciones Unidas sobre los derechos de los pueblos indigenas, art. 32, num. 3.

17 ONU, Declaración de las Naciones Unidas sobre los derechos de los pueblos indígenas, art. 30, num. 1. 
el conflicto armado se ha intensificado en los años recientes. Las confrontaciones entre los actores es una situación cotidiana que causa desplazamiento, asesinatos, retenciones colectivas, secuestros, desapariciones, ejecuciones extrajudiciales, heridas y lesiones personales. Ante estos hechos que vulneran la convivencia en los territorios considerados ancestrales, las reclamaciones por parte de las organizaciones políticas de las comunidades indígenas frente a los Gobiernos locales y nacionales no han sido escuchadas; el conflicto continúa su complejo curso y deja víctimas y victimarios en los territorios.

La pretensión de que las fuerzas militares estatales adelanten la consulta previa con las comunidades indígenas llevaría a que las hostilidades armadas se minimicen, se reduzca la intensidad del conflicto y a que organizaciones propias y locales, como las guardias indígenas, sean las responsables de la convivencia en los resguardos.

Otro documento internacional que incorpora la consulta previa para los pueblos indígenas es la reciente Declaración americana sobre los derechos de los pueblos indígenas, del 14 de junio de 2016. Expone la consulta para medidas legislativas y administrativas que involucren a las comunidades: "2. Los Estados celebrarán consultas y cooperarán de buena fe con los pueblos indígenas interesados por medio de sus instituciones representativas antes de adoptar y aplicar medidas legislativas o administrativas que los afecten, a fin de obtener su consentimiento libre". ${ }^{18} \mathrm{El}$ único Estado de la región que se distanció fue el colombiano: "El Estado de Colombia se aparta del consenso respecto del artículo XXIII, numeral 2, de la Declaración de los Pueblos Indígenas de la OEA, que se refiere a las consultas". ${ }^{19}$

La Declaración de las Naciones Unidas sobre los derechos de los campesinos y de otras personas que trabajan en las zonas rurales, del 17 de diciembre de 2018, impulsa el reconocimiento de derechos de las comunidades campesinas y otros colectivos rurales; sin embargo, las abstenciones por América Latina correspondieron a Argentina, Brasil y Colombia, pese a tener una representativa población campesina y rural: "[...] la adopción se formalizó por parte de la Asamblea General el 17 de diciembre con 121 votos a favor, 54 abstenciones y 8 votos en contra". ${ }^{20}$

18 Organización de los Estados Americanos [OEA], Declaración americana sobre los derechos de los pueblos indigenas (Washington, 14 de junio de 2016), art. 24, num. 2.

19 OEA, Declaración americana sobre los derechos de los pueblos indígenas, art. 24, num. 3. Nota al pie.

20 Red Nacional de Agricultura Familiar, "La declaración de los derechos de los campesinos sí podría proteger al campesinado colombiano", https://agriculturafamiliar.co/la-declaracion-de-derechos-campesinos-si-podriaproteger-al-campesinado-colombiano/ (acceso mayo 25, 2021). 
Esta Declaración complementa el compendio de documentos internacionales que promueven la consulta previa como un derecho que pretende proteger a las comunidades diferenciadas: "Los Estados celebrarán consultas y cooperarán de buena fe con los campesinos [...] dialogando con quienes puedan verse afectados por las decisiones [...] asegurando una participación activa, libre, efectiva, significativa e informada de las personas". ${ }^{21}$

El reconocimiento que se ha brindado desde las declaraciones sobre los pueblos indígenas y las comunidades ha impedido que sus territorios sigan siendo apropiados por intereses particulares y transnacionales de una forma arbitraria y avasalladora. Para que estos tratados sean llevados a buen término, las ONG internacionales han sido determinantes, con su acompañamiento en terreno y con las redes comunitarias y de expertos, también de origen transnacional, que adelantan acciones de protección:

[...] debido a la expansión de empresas transnacionales y multinacionales en territorios de comunidades indígenas los conflictos se han acentuado [...] ha llevado a la configuración de alianzas y relaciones internacionales con ONG de derechos humanos y de protección de las poblaciones originarias. ${ }^{22}$

\section{Los avances de la Corte Constitucional colombiana en consulta previa}

El derecho a la consulta previa ha sido paulatinamente desarrollado en el contexto colombiano por las decisiones de la Corte Constitucional. Un referente emblemático fue la Sentencia SU-039 de 1997, que lo impulsó desde lo jurisprudencial:

[... ] participación de las comunidades indígenas en las decisiones que pueden afectarlas en relación con la explotación de los recursos naturales [...] a través del mecanismo de la consulta, adquiere la connotación de derecho fundamental [...] para preservar la integridad étnica. ${ }^{23}$

21 Organización de las Naciones Unidas [ONU], Declaración de las Naciones Unidas sobre los derechos de los campesinos y de otras personas que trabajan en las zonas rurales (Nueva York, 17 de diciembre de 2018), art. 2 , num. 3.

22 Jairo Vladimir Llano Franco, "Pluralismo jurídico, diversidad cultural, identidades, globalización y multiculturalismo: perspectiva desde la ciencia jurídica", Novum Jus 10, núm. 1 (2016): 72, DOI: https:// doi.org/10.14718/NovumJus.2016.10.1.3 (acceso septiembre 8, 2021).

23 República de Colombia, Corte Constitucional, Sentencia SU-039 de 3 de febrero de 1997, M. P. Antonio Barrera Carbonell. 
Para ese momento, los pueblos indígenas no compartían la consulta previa, ya que la miraban como una intromisión en sus territorios por personas externas que tenían unos intereses, como apropiarse de sus recursos. ${ }^{24}$ Dicha percepción se presentó en varias deliberaciones con las comunidades que la rechazaban y consideraban que, en vez de beneficiarse, les afectaba negativamente:

[Para] algunos miembros del movimiento indígena colombiano los procesos de consulta previa no han tenido buenos resultados. Argumentan que han sido acompañados los procesos de múltiples, sistemáticas y complejas violaciones de los derechos humanos y especialmente el derecho a la vida. ${ }^{25}$

Esta prevención se mantuvo por varios años. En el año 2008, en el curso-encuentro "Consulta previa: perspectiva crítica de las experiencias y aportes a su consolidación", paralelo al VI congreso de la Red Latinoamericana de Antropología Jurídica (Relaju) en Bogotá, participaron representantes de diversas comunidades indígenas del país. Allí se expusieron las reservas ante esta, debido a la percepción de las comunidades de que las beneficiadas eran las empresas y las instituciones estatales, y se reproducía la exclusión socioeconómica y cultural. ${ }^{26}$

Paulatinamente, en discusiones entre las mismas comunidades indígenas, las reflexiones con expertos provenientes de la antropología jurídica, la sociología y la filosofía del derecho, la academia en su conjunto y las entidades estatales, así como por la jurisprudencia de la Corte Constitucional, las comunidades comenzaron a mirarla como un derecho que les traería más beneficios que afectaciones negativas: "Colombia ha construido un ámbito de reflexión interdisciplinaria entre abogados, antropólogos, filósofos, matemáticos, lógicos y personas de las comunidades étnicas, muy reconocido a nivel internacional que se denomina otro derecho". ${ }^{27}$

24 La suspicacia tiene, no obstante, bastante fundamento en la realidad, pero también expresa una preocupación que ha convocado la acción del movimiento indígena, Tania Giovanna Vivas-Barrera, Alejandro Quintero Sánchez y Bernardo Pérez Salazar, "Propiedad colectiva de la tierra y movimiento indígena en América Latina", Opción 35, núm. 25 esp. (2019): 1323-1354.

25 Esther Sánchez Botero, "El aporte de la antropología jurídica a la construcción del pluralismo jurídico legal y oficial: ¿ficción persuasiva?" en El peritaje antropológico como prueba judicial, eds. Esther Sánchez Botero y Herinaldy Gómez (Bogotá: Red Latinoamericana de Antropología Jurídica (Relaju), 2018), 89.

26 Jairo Vladimir Llano Franco, "Reconocimiento cultural y exclusión socioeconómica. Resumen", VI Congreso de la Red Latinoamericana de Antropología Jurídica Relaju. Diversidad étnica y cultural: desarrollos constitucionales, políticas y prácticas nacionales (Bogotá: Red Latinoamericana de Antropología Jurídica, 2008), 3031

27 Esther Sánchez Botero, "Algunas reflexiones sobre la consulta previa en Colombia" en Consulta previa, principios, enfoque metodológico e instrumentalización. Experiencias compartidas y lecciones aprendidas (Bogotá: Red Latinoamericana de Antropología Jurídica Relaju, 2008), 28. 
Lo paradójico es que, en la actualidad, son las comunidades indígenas las que se apropiaron del derecho a la consulta hasta el punto de que por medio de la acción de tutela están pidiendo que les sea protegido. Ya no se percibe como una intromisión, sino como acción protectora de sus territorios. Este avance encuentra acompañamiento en las decisiones de la Corte Constitucional en las que ha reiterado que la tutela procede si la interponen las mismas comunidades o sus representantes cuando se percaten de la vulneración. Para el Tribunal:

[La] existencia de mecanismos ordinarios de defensa judicial no excluye la interposición de la tutela para efectos de amparar el derecho a la consulta previa Adicionalmente, la Corte Constitucional ha considerado que la tutela es el mecanismo de defensa judicial idóneo. ${ }^{28}$

También es contradictorio lo que sucedió con los empresarios y los representantes de las entidades estatales, pues, en un primer momento, la aceptaban, debido a que se convertía en una opción para implementar sus proyectos de intervención en los territorios, por supuesto, con el consentimiento comunitario, pero la percepción se transformó; el rechazo proviene de los sobrecostos que originan los procesos de consulta y la implementación de los acuerdos por medio de compensaciones; además, los funcionarios consideran que este derecho es un obstáculo para el desarrollo socioeconómico en el país:

En los años recientes, se ha planteado un desmonte muy sutil de los derechos de los pueblos indígenas, debido a que sectores conservadores de las élites políticas y económicas han cuestionado la autonomía cultural y jurídica que la Corte ha ampliado. ${ }^{29}$

Las pretensiones de limitación del derecho a la consulta previa por parte de los mismos funcionarios gubernamentales y las instituciones estatales estaría en contravía de lo que caracteriza a un Estado constitucional y democrático de derecho, donde el deber de estos entes es garantizar que los derechos sean cumplidos en la mayor medida posible para beneficio de los ciudadanos, en este caso, las comunidades que además tienen una especial protección constitucional, la cual ha sido determinada

28 República de Colombia, Corte Constitucional, Sentencia T-063 de 15 de febrero de 2019, M. P. Antonio José Lizarazo Ocampo.

29 Jairo Vladimir Llano Franco, "Pluralismo jurídico y desmonte de derechos en comunidades étnicas de Brasil y Colombia" en Desigualdades y conflictos en el Pacífico colombiano, comp. Jairo Vladimir Llano Franco (Bogotá: Ibáñez, 2020), 193. 
por la Corte Constitucional: "[... ] esta Corporación ha sido clara al señalar que los pueblos indígenas y las comunidades negras, afrodescendientes o afrocolombianas son titulares de derechos fundamentales y sujetos de especial protección constitucional". ${ }^{30}$

A las comunidades indígenas y negras, que gozan de especial protección constitucional, se les suman las campesinas: "La jurisprudencia de esta Corporación ha considerado que los campesinos y los trabajadores rurales son sujetos de especial protección constitucional en determinados escenarios. Lo anterior, atendiendo a las condiciones de vulnerabilidad y discriminación que los han afectado históricamente". ${ }^{31}$

Pese a las tensiones descritas, la implementación de este derecho avanza, debido a que tiene un aval sociocultural que lo posiciona en el país y la región, porque parte del diálogo intercultural entre diferentes que pocas veces se encuentran y se logra por medio de proyectos con los que se pretende incrementar el bienestar de todos, unos desde lo empresarial y otros desde las tradiciones: "Las interacciones de los colectivos humanos son permanentes y constantes en estas últimas décadas [...] la se convierte en la forma más plausible de las relaciones entre las culturas, las sociedades y los individuos". 32

Para casos concretos en los que se lleva a cabo la consulta previa en el contexto colombiano, el diálogo intercultural surge cuando las entidades lo proponen a causa de sus múltiples proyectos de intervención. Las comunidades que poco a poco se lo apropian y lo convierten en un referente para la reproducción cultural y la protección de la diversidad ante el desarrollo y el progreso socioeconómico. Este diálogo intercultural ha permitido que la consulta sea implementada mediante: "[...] diálogos interculturales en los cuales se pasa de tensiones y confrontaciones a arreglos que solidifican relaciones entre estas culturas". ${ }^{33}$

Pero la interculturalidad no se plasma solo en la consulta previa; también es un espacio donde el pluralismo jurídico se lleva a cabo: "El pluralismo [...] justifica el derecho a la consulta previa apelando a los derechos del autogobierno y la

30 República de Colombia, Corte Constitucional, Sentencia T-414 de 2 de julio de 2015, M. P. Luis Guillermo Guerrero Pérez.

31 República de Colombia, Corte Constitucional, Sentencia C-077 de 8 de febrero de 2017, M. P. Luis Ernesto Vargas Silva.

32 Llano Franco, "Pluralismo jurídico", 22.

33 Jairo Vladimir Llano Franco, Teoría del Estado y del derecho. Pluralismo jurídico (Bogotá: Ibáñez, 2017), 495. 
integridad cultural, además de justificarlo en razones de igualdad intercultural y justicia correctiva [...] vinculado con una interpretación pluralista del Estado". ${ }^{34}$

Una representación de pluralismo en el espacio de la consulta previa se presenta porque este proviene del derecho internacional público. El Convenio 169 de la OIT de 1989, que fue el primer documento internacional que lo plasmó, fue recogido por el derecho estatal por medio de la Ley 21 de 1991 y la jurisprudencia de la Corte Constitucional en distintas sentencias desde finales de la década de 1990 hasta el presente. De esta manera, la protección y la garantía concurren en un solo espacio, por medio del derecho internacional y global, el derecho estatal, el derecho de usos y costumbres de las comunidades indígenas: "El pluralismo jurídico debe ser visualizado como el esfuerzo teórico de explicar la convivencia contradictoria, unas veces consensual y otras veces conflictivo, entre los varios derechos observables en una misma sociedad". ${ }^{35}$

\section{Consulta previa y proyectos viales}

La consulta previa es procedente en los proyectos de infraestructura vial que afectan territorios de comunidades indígenas, afros y tribales, ya que el impacto sociocultural de este tipo de intervenciones no se limita al territorio afectado, pues transforma la cultura de una determinada comunidad, debido a que estos proyectos son perdurables. La vía que se construye implica una convivencia con ella por varias décadas.

La Corte Constitucional determinó que, en proyectos viales que pasen por territorios de comunidades, se hace necesaria la consulta previa; mientras no se realice, las obras quedan suspendidas, como sucedió con un plan que afectaba a la comunidad indígena de los embera-katío en el departamento del Chocó: "La consulta previa y la búsqueda del consentimiento informado deberán observarse bajo [...] garantías descritas en esta providencia [...] se ordenará suspender la ejecución de las obras de la carretera denominada Unguía-Acandí ó Titumate-Balboa-San Miguel-Acandî". ${ }^{36}$

34 Daniel Bonilla Maldonado, "Autogobierno e identidad cultural. La Corte Constitucional colombiana y el derecho a la consulta previa" Constitucionalismo del Sur Global, ed. Daniel Bonilla Maldonado (Bogotá: Siglo del Hombre Editores, 2015), 312.

35 Antonio Carlos Wolkmer, Pluralismo jurídico. Fundamentos de uma nova cultura no direito, 3a ed. (San Paulo: Alfa Omega, 2001), 255.

36 República de Colombia, Corte Constitucional, Sentencia T-129 de 3 de marzo de 2011, M. P. Jorge Iván Palacio Palacio. 
En el proceso de reconocer la consulta previa ante proyectos viales, la Corte Constitucional amplió la cobertura a los sitios sagrados y espirituales que se encuentran por fuera y en frontera de sus territorios y señaló que: "[...] proyectos de infraestructura vial tienen la posibilidad de afectar de manera directa a las comunidades [...] las colectividades deben ser consultadas sobre esas medidas [...] adquieren importancia los lugares sagrados que no se encuentran al interior de los resguardos". ${ }^{37}$

De acuerdo con la experiencia en consulta previa desde la asesoría a las comunidades y la investigación, las siguientes son las fases y afectaciones que conllevan un proyecto vial.

Primera fase: construcción de la vía

Conlleva afectación del paisaje e impacto ambiental considerable. Los sitios espirituales se desplazan, la movilidad tradicional se adecúa a la que se propone con la vía, la comunicación entre la comunidad se limita, las viviendas tradicionales y los sitios de encuentros culturales son reubicados y la alimentación tradicional se ve menoscabada, entre otras situaciones que alteran la vida sociocultural.

Segunda fase: utilización de la vía

Beneficia a los vehículos y no a los peatones, se produce contaminación ambiental y un choque cultural por la presencia permanente de externos a la comunidad propia, surgen procesos de aculturación constante y la calidad de vida se ve afectada. La primera se mantiene durante los años de construcción y la segunda, por décadas.

Ante estos efectos, la consulta previa se vuelve indispensable para beneficio de las partes, ya que son obras que perduran. La consulta no va en contravía de alguno de los participantes del proceso, sino que pretende tanto la protección de las comunidades como el mantenimiento del proyecto desde la perspectiva del diálogo intercultural. Al adelantar una aceptable consulta se evitan conflictos y tensiones desde el inicio hasta la fase final.

37 República de Colombia, Corte Constitucional, Sentencia T-436 de 12 de agosto de 2016, M. P. Alberto Rojas Ríos. 
Según el derecho internacional de los derechos humanos, los proyectos viales deben consultarse siempre que se construyan en territorios de comunidades indígenas, negras o campesinas, debido a que son estrategias para el desarrollo socioeconómico de interés general y de particulares, que alteran sus recursos naturales para beneficio colectivo. Ante esta situación, la pretensión es lograr el consentimiento para las intervenciones y posibilitar una compensación socioeconómica, cultural y moral por las afectaciones que estas ocasionen, complementado con medidas que lleven a la mitigación de sus consecuencias nocivas. Estas medidas, por lo general, surgen del mismo proceso de implementación de la consulta previa.

\subsection{Proyecto vial doble calzada Popayán-Santander de Quilichao}

La necesidad de ampliar la vía Panamericana, una de las más importantes en el aspecto socioeconómico, debido a que comunica los departamentos del sur del país y los países fronterizos (Ecuador y Perú) con las ciudades del occidente (Cali) y del centro (Bogotá y Medellín), ha llevado a los gobiernos nacional, departamentales y locales a impulsar su transformación. Con ello se pretende mejorar la conexión y dinamizar los intercambios internacionales y locales, para ampliar las relaciones económicas: "Entre enero y noviembre del año 2013, las exportaciones de Ecuador a Colombia fueron de US $\$ 801,82$ millones de dólares, mientras que las importaciones en el mismo período, de Ecuador provenientes de Colombia llegaron a US\$ 1804,67 millones de dólares". ${ }^{38}$

Esto llevó a que se creara una alianza público-privada para convertir la vía PopayánSantander de Quilichao en doble calzada; el responsable de la ejecución es el consorcio Nuevo Cauca que, en su misión, expresa las actividades que desarrollará: "Prestar a los usuarios del corredor vial Popayán-Santander de Quilichao, servicios adecuados y oportunos que garanticen la integridad, comodidad y seguridad vial [...] y construcción de la segunda calzada, su operación y mantenimiento hasta la reversión del proyecto". ${ }^{39}$

Este proyecto de intervención que trae beneficios para particulares, empresarios locales y transnacionales — que además son los promotores del comercio e

38 Martha Lucía Márquez Restrepo, "Colombia, Venezuela y Ecuador: cooperación y coexistencia de cara al posacuerdo" en Política exterior colombiana: escenarios y desafios en el posconflicto, ed. Eduardo Pastrana Buelvas y Hubert Gehrin (Bogotá: Pontificia Universidad Javeriana, 2015), 556.

39 Consorcio Nuevo Cauca, "Misión y visión", https://www.nuevocauca.com/mision-y-vision/ (acceso 8 de mayo, 2021). 
intercambio económico internacional y nacional - tiene en las entidades estatales y sus funcionarios un apoyo determinante, soportado en la concepción de desarrollo y progreso socioeconómico para el país y en la idea de interés general. Lo complejo de este proyecto vial es que se construirá en una región donde la diversidad cultural es predominante y deben ser trazadas en límites o en los territorios de los pueblos indígenas y las comunidades negras y campesinas del departamento del Cauca.

Esta amplia diversidad cultural del Departamento se refiere a los pueblos indígenas que se encuentran allí instalados, como los nasa, misak, yanacona, embera, inga y coconuco, que poseen sus respetivos resguardos y tienen una población significativa:

[...] la población indígena en el reciente censo de 2018 en el Cauca fue de 308455 habitantes [...] este dato contrasta en el mismo informe con los 351419 de la cartografía social y otros censos indígenas realizados en el $2017 .{ }^{40}$

Las comunidades negras tienen sus territorios por medio de los consejos comunitarios en el norte, el Pacífico y el sur del departamento, así como una notable población se encuentra organizada en zonas de reservas campesinas, cooperativas y agremiaciones. Esta compleja diversidad étnica lleva a que la mayoría de la población del departamento se encuentre en las zonas rurales: "El 39,85 \% de la población del departamento se ubica en la zona urbana y el 60,15 \% en la zona rural". ${ }^{41}$

Ante este contexto sociocultural, se hace necesario adelantar las consultas previas desde una perspectiva intercultural, que permitan llegar a un acuerdo para materializar este proyecto con las menores afectaciones posibles y para que se convierta en una posibilidad de inclusión; "un diálogo fundamentado en la interrelación cuyo fin no es la superposición de una cultura sobre otra, sino el crecimiento mutuo por medio de los cambios [...] para la construcción de una existencia mejor por medio del diálogo intercultural". ${ }^{42}$

En este complejo espacio de diversidad, cualquier proyecto de intervención requiere un diagnóstico detallado de la población a la que afectará, pues, de no hacerse

40 Juliana Zapata Galvis y Jairo Vladimir Llano Franco, "Acuerdo de paz, territorio y diversidad en el norte del Cauca" en Pos-acuerdo y territorio en las comunidades indigenas, afro y campesinas en el norte del Cauca, eds. Jairo Vladimir Llano Franco et al. (Bogotá: Ibáñez, 2019), 54.

41 Gobernación del Cauca, "Plan departamental de desarrollo 2016-2019. Cauca territorio de paz", http:// anterior.cauca.gov.co/informes/plan-desarrollo (acceso mayo 10, 2021), 24.

42 Antonio Carlos Wolkmer, Teoría crítica del derecho desde América Latina (Ciudad de México: Akal, 2017), 164. 
en terreno podría desconocerse alguna de las comunidades reconocidas desde los postulados constitucionales: "[...] para considerar que existe una etnia deben identificarse en un grupo humano dos condiciones: 1) una subjetiva a lo que se ha llamado conciencia étnica [...] 2) una objetiva, que se refiere a los elementos materiales que distinguen al grupo". ${ }^{43}$

Reducir la presencia de comunidades diferenciadas a lo que determine el Ministerio del Interior por medio de una certificación, es limitante para un proyecto de intervención. La diversidad cultural supera ciertos formalismos estatales y se encuentra en los territorios del país; además, en los documentos que forman parte del derecho internacional acerca de la consulta previa, en ningún momento se solicita que, para existir en un determinado territorio, las comunidades necesitan la certificación de un Ministerio perteneciente al Gobierno central. Las comunidades se localizan y reproducen sus cosmovisiones como parte de su identidad cultural, que está reconocida como un derecho soportado en los principios de identidad y cultura, pues:

[El] derecho a la identidad refiere al resguardo del sentido de pertenencia que un individuo tiene respecto de una determinada cultura. Y, por otra parte, este derecho fundamental apunta a la protección de la cultura a la que pertenece el sujeto. ${ }^{44}$

El reconocimiento de los principios de identidad y cultura para beneficio de los pueblos que forman parte del Estado y de la sociedad colombiana se ha logrado a partir de la Constitución y de la interpretación que hacen los jueces constitucionales sobre la diversidad cultural, aunque son las mismas comunidades las que definen la existencia del grupo étnico: "En la Sentencia T-576 de 2014 se aclaró que no son las autoridades administrativas ni judiciales llamadas a establecer si una comunidad étnica 'existe', si es 'étnicamente diversa' o si determinado individuo pertenece [...]. Ello corresponde a las propias comunidades". ${ }^{45}$

43 Gloria Amparo Rodríguez, De la consulta previa al consentimiento libre, previo e informado a pueblos indígenas en Colombia, 2a ed. (Bogotá: Universidad del Rosario Ibáñez, 2017), 63.

${ }^{44}$ Juan Jorge Faundes Peñafiel y Silvina Ramírez, "El derecho a la identidad cultural, horizontes plurales latinoamericanos" en Derecho fundamental a la identidad cultural: abordajes plurales desde América Latina, eds. Juan Jorge Faundes Peñafiel y Silvina Ramírez (Santiago: Universidad Autónoma de Chile, 2020), 21.

45 República de Colombia, Corte Constitucional, "Diversidad y pluralismo", https://www.corteconstitucional. gov.co/Transparencia/publicaciones/pluralismo\%202020\%20v3\%2011220.pdf (acceso abril 30, 2021), 34 . 
La Gobernación del departamento del Cauca, en sus documentos institucionales como el más reciente plan de desarrollo, ha expuesto la densidad de la población indígena, pues se encuentran " 110 resguardos indígenas ubicados en 30 municipios y 86 comunidades asentadas por fuera de los resguardos", ${ }^{46}$ incluido Caldono con presencia significativa nasa, con sus resguardos y cabildos:

[...] el departamento cuenta aproximadamente con 270000 personas distribuidas en todo el territorio caucano, concentrándose principalmente en la subregión norte, donde habita el 43 \% de esta población. Los municipios con mayor número de población indígena son Toribío con 25425 y Caldono con $23385 .{ }^{47}$

El plan de desarrollo del Cauca muestra al municipio de Caldono, en la zona centro-norte, entre los de mayor población indígena del departamento; lo contradictorio es que no fue consultada por el consorcio nuevo cauca. Por lo menos debió solicitar un diálogo inicial con las comunidades, primero, para presentarles el proyecto y, segundo, para mirar las posibilidades de llevar a cabo un proceso de consulta previa.

Este proyecto vial afectaría dos resguardos pertenecientes al pueblo nasa en los municipios de Caldono y, en menor medida, de Piendamó. Ante la ausencia de consulta previa por parte del consorcio, las comunidades decidieron interponer acción de tutela que, en la instancia final, fue seleccionada y resuelta por la Corte Constitucional en el Auto 65 de 2018:

Las comunidades indígenas "La Laguna Siberia" y "Las Mercedes", a través de dos de sus autoridades indígenas, N. P. C. y N. M. C. G., consideran que el Ministerio del Interior, el Ministerio de Transporte, la Agencia Nacional de Infraestructura (en adelante ANI), la Autoridad Nacional de Licencias Ambientales (en adelante ANLA) y Nuevo C. S. comprometen su derecho fundamental a la consulta previa por haber desarrollado el proyecto vial "Segunda Calzada Popayán-Santander de Quilichao", y concretamente su unidad funcional 3, sin haberles consultado. ${ }^{48}$

46 Gobernación del Cauca, "Plan de desarrollo departamental 2020-2023. Motivos para avanzar", https://www. cauca.gov.co/NuestraGestion/PlaneacionGestionyControl/Plan\%20de\%20Desarrollo\%20Departamental\%20 2020\%20-\%202023.pdf (acceso mayo 7, 2021).

47 Gobernación del Cauca, "Plan departamental de desarrollo 2016-2019".

48 República de Colombia, Corte Constitucional, Auto 651 de 8 de octubre de 2018, Expediente T-6823931, M. P. Gloria Stella Ortiz Delgado. 
La Corte Constitucional confirmó que los dos resguardos se componen de veredas que se encuentran al lado de la vía Panamericana. El de la Laguna Siberia, en su ampliación territorial, tiene una incidencia determinante de la vía:

El resguardo indígena Nasa "La Laguna Siberia" [... ] ha sido ampliado mediante la Resolución 039 de 2003 [... . con 10522 hectáreas en las veredas de Cabuyal, Panamericana, La Buitrera, Pescador, P. y Puente Real, que son atravesadas por la vía Panamericana. ${ }^{49}$

Por su parte, el resguardo Las Mercedes, que tiene un reconocimiento más reciente, también presenta veredas que limitan con la vía a intervenir:

El resguardo indígena Nasa "Las Mercedes" [... ] constituido por la Resolución 003 del 22 de julio de 2003 por el Ministerio del Interior. Se asientan en varias veredas como Monterilla y el Pital que también son atravesadas por la vía Panamericana. ${ }^{50}$

Parte de estos territorios serían intervenidos por parte del consorcio Nuevo Cauca con el proyecto vial doble calzada Santander de Quilichao-Popayán. Ante esta situación, se debe gestionar la consulta previa con la comunidad indígena nasa, debido a que el proyecto altera de forma directa su territorio y afectaría su identidad cultural. Adelantar la consulta no significa que el proyecto se cancele, sino que se evalúa colectivamente con los pueblos de la zona, los cuales serían compensados si se modifican de forma ostensible su cultura y sus espacios socioculturales.

Este requisito de llevar a cabo la consulta previa con el pueblo nasa de los resguardos La Laguna Siberia y Las Mercedes no fue cumplido de forma directa y lo que se protegió fue el debido proceso, en respuesta a la acción de tutela, por parte de la Corte Constitucional:

[...] la Sala enfatizó en que, si bien la Defensoría del Pueblo constató cierta proximidad de los miembros de dichos grupos con la Vía Panamericana, la falta de información sobre el proyecto impide que los miembros de la comunidad y, a través de ellos la Defensoría y esta Corporación, se hagan una idea cierta del impacto del mismo [...]. Por lo tanto, no protegerá el derecho a la consulta

\footnotetext{
49 República de Colombia, Corte Constitucional, Auto 651.

50 República de Colombia, Corte Constitucional, Auto 651.
} 
previa en forma directa, sino que lo hará a través de la protección al debido proceso, en el sentido ya referido en los fundamentos jurídicos anteriores. ${ }^{51}$

Ante la orientación de la Corte Constitucional, la Dirección de Consulta Previa del Ministerio del Interior hizo una visita de campo e interdisciplinaria e interpretó que el proyecto vial no afectaba alguno de los resguardos, porque se encontraban un par de kilómetros por fuera del perímetro del proyecto vial y que en este espacio territorial las prácticas culturales del pueblo nasa no se llevaban a cabo. Esto quedó plasmado en la Resolución 114 del 27 de noviembre de 2014, en la cual solo se incluye el resguardo Las Mercedes:

[...] el resguardo Las Mercedes ni su comunidad no va a ser alterado por el proyecto, en tanto que las prácticas colectivas de la comunidad se realizan en su mayoría dentro del territorio geográfico. Adicionalmente, el Resguardo Las Mercedes se encuentra en una distancia geográfica de 2,89 kilómetros con relación al proyecto. ${ }^{52}$

Esta Resolución tiene varias apreciaciones que estarían en contravía de los estudios y análisis sobre comunidades indígenas. Las instituciones estatales determinan hasta dónde llega el territorio desde la perspectiva cultural, aunque los que definen los límites territoriales y culturales son los mismos pueblos. Las fronteras no son formales en comunidades ancestrales. En su proceso de reproducción cultural se les adjudican territorios para que puedan desenvolverse desde su identidad; por eso, decir que los más de 2 kilómetros que separan el resguardo de la vía que se construiría y justifica que no haya consulta previa es desfasado, no solo en términos territoriales, sino también culturales. Dos o tres kilómetros es poco y en una geografía quebrada como la de las laderas caucanas, puntualizarlo es más complejo aún. En el aspecto territorial el argumento de los 2 kilómetros beneficia a la comunidad, pero el cultural, un límite de este tipo causa ruptura con los territorios ancestrales y de identidad cultural que se han construido por décadas.

Para la decisión de la tutela, la Corte Constitucional consultó a distintas universidades para que emitieran sus conceptos académicos: los Departamentos de Antropología, de la Universidad Nacional y la de los Andes, los programas de Derecho y grupos de investigación pertenecientes a las Universidades Católica, Libre, Tecnológica del

51 República de Colombia, Corte Constitucional, Sentencia T-281 de 20 de junio de 2019, M. P. Gloria Stella Ortiz Delgado.

52 República de Colombia, Ministerio del Interior, Resolución 114 de 2019 (Bogotá, 27 de noviembre de 2019). 
Chocó y Externado de Colombia. Sin tener contacto alguno, se observaron puntos de encuentro en sus conceptos; uno de los principales fue el de llevar a cabo la consulta previa con el pueblo nasa en los resguardos Las Mercedes y La Laguna Siberia, pues "la consulta previa es una expresión del carácter democrático y pluralista [...] es necesaria la consulta previa en la medida en que la vía Panamericana atraviesa las veredas en las que los resguardos demandantes se asientan". ${ }^{53}$

El otro punto consiste en afirmar que el hecho de que este proyecto vial se encuentre a un par de kilómetros de los resguardos afecta a la comunidad, por lo cual sería necesario adelantar y garantizar el proceso consultivo con los indígenas.

El propósito es que las comunidades indígenas no sean perturbadas ni se les impongan proyectos de desarrollo, con la pretensión de que logren conservar sus cosmovisiones. También es claro que las culturas aisladas son inexistentes, pero esa relación intercultural debe ser soportada por un diálogo en el que los beneficiados, en un primer momento, sean las comunidades; en segundo lugar, las partes, y en tercer término el mismo proyecto; por eso, la consulta previa se convierte en un derecho protector, igualitario y democrático.

\section{Conclusiones}

Las deliberaciones contemporáneas sobre el derecho fundamental a la consulta previa se encuentran entre quienes proponen su limitación, en concordancia con los intereses de las entidades estatales y el empresariado que adelanta proyectos de intervención en los territorios étnicos, y quienes consideran que la consulta previa debe ampliarse y consolidarse, en armonía con las recientes declaraciones internacionales de derechos sobre la diversidad y las comunidades étnicas; entre los últimos se encuentran las organizaciones políticas de pueblos indígenas y comunidades negras y campesinas, sectores académicos, ONG y gobernantes que pretenden impulsar el diálogo intercultural.

Dicho diálogo se ha convertido en prioritario, puesto que las comunidades se han convertido en referentes para el cuidado ambiental. En el contexto colombiano, estas comunidades se ubican en zonas de páramo y de reserva natural, donde se hallan los nacimientos de agua, producto esencial para el sostenimiento de la humanidad, entre una amplia diversidad de flora y fauna, que necesita cuidado para mantener

53 República de Colombia, Corte Constitucional, Sentencia T-281. 
esta biodiversidad; por eso, garantizar el derecho a la consulta previa permitirá la protección no solo de los pueblos, sino también de los recursos naturales que los rodean.

\section{Referencias}

Barreto, José-Manuel. "Decolonial strategies and dialogue in the human rights field: A manifesto", Transnational Legal Theory 3, núm. 1 (2012): 1-29.

Barreto, José-Manuel. "Decolonial thinking and the quest for decolonising human rights". Asian Journal of Social Science 46, núm. 4-5 (2018): 484-502.

Bonilla Maldonado, Daniel. "Autogobierno e identidad cultural. La Corte Constitucional colombiana y el derecho a la consulta previa" en Constitucionalismo del Sur Global, editado por Daniel Bonilla Maldonado, 305-358. Bogotá: Siglo del Hombre, 2015.

Camacho Bustamante, Aracelis Isabel. "Las leyes de justicia y paz, de reparación y restitución de tierras y sus efectos en la sociedad colombiana". Nuevos Paradigmas de las Ciencias Sociales Latinoamericanas 10, núm. 20 (2019): 85-94.

Carvajal-Martínez, Jorge Enrique. La sociología jurídica en Colombia. Bogotá: Universidad Libre, 2016.

Consorcio Nuevo Cauca. "Misión y visión". https://www.nuevocauca.com/mision-y-vision/ (acceso 8 de mayo, 2021).

Costa, Pietro. "Ciudadanía y patrones de pertenencia a la comunidad política" en Nacionalidad y ciudadanía, editado por Pietro Costa y Benito Aláez Corral, 19-48. Madrid: Fundación Coloquio Jurídico Europeo, 2010.

Cruz Chavarro, Nasly Nayely y Gabriel Andrés Arévalo Robles. "Cumbres indígenas: política y diplomacia ancestral en América Latina". Novum Jus 15, núm. 1(2021): 133-160, DOI: https://doi.org/10.14718/NovumJus.2021.15.1.7 (acceso noviembre 10, 2021).

Cubides-Cárdenas, Jaime Alfonso, Paola Alexandra Sierra-Zamora, Diego Alexander Calixto Ortiz y Nicolás Pabón-Caballero. "Terrorismo por parte de las FARC-EP y políticas públicas orientadas a la seguridad nacional en Colombia durante 1990-2000". Revista Científica General José María Córdova 17, núm. 26 (2019): 308-325.

Dávila, Luis Felipe y Caroline Doyle. "Insider and outsider fieldwork challenges in Medellín, Colombia". International Journal for Crime, Justice and Social Democracy 9, núm. 3 (2020): 87-99.

Faundes Peñafiel, Juan Jorge y Silvina Ramírez. "El derecho a la identidad cultural, horizontes plurales latinoamericanos" en Derecho fundamental a la identidad cultural: abordajes plurales desde América Latina, editado por Juan Jorge Faundes Peñafiel y Silvina Ramírez, 13-32. Santiago: Universidad Autónoma de Chile, 2020. 
Gobernación del Cauca. "Plan de desarrollo departamental 2020-2023. Motivos para avanzar". https://www.cauca.gov.co/NuestraGestion/PlaneacionGestionyControl/ Plan\%20de\%20Desarrollo\%20Departamental\%202020\%20-\%202023.pdf (acceso mayo 7,2021 ).

Gobernación del Cauca. "Plan departamental de desarrollo 2016-2019. Cauca territorio de paz". http://anterior.cauca.gov.co/informes/plan-desarrollo (acceso mayo 10, 2021).

González Monguí, Pablo Elías. "La negación de la calidad de ciudadano o de persona en el derecho penal de enemigo", Opción 35, núm. 25 esp. (2019): 1070-1103.

González Monguí, Pablo Elías. "La selectividad penal negativa de las muertes ilegítimamente presentadas como bajas en combate por la fuerza pública del Estado colombiano". Revista Latinoamericana de Sociología Jurídica 1, núm. 1 (2020): 305-345.

Llano Franco, Jairo Vladimir y Germán Silva García. "Globalización del derecho constitucional y constitucionalismo crítico en América Latina”. Utopía y Praxis Latinoamericana 23, núm. 2 extra (2018): 59-73.

Llano Franco, Jairo Vladimir. "Pluralismo jurídico y desmonte de derechos en comunidades étnicas de Brasil y Colombia" en Desigualdades y conflictos en el Pacífico colombiano, compilado por Jairo Vladimir Llano Franco, 177-207, Bogotá: Ibáñez, 2020.

Llano Franco, Jairo Vladimir. "Pluralismo jurídico, diversidad cultural, identidades, globalización y multiculturalismo: perspectiva desde la ciencia jurídica”. Novum Jus 10, núm. 1 (2016): 49-92, DOI: https://doi.org/10.14718/NovumJus.2016.10.1.3 (acceso septiembre 8, 2021).

Llano Franco, Jairo Vladimir. "Reconocimiento cultural y exclusión socioeconómica". Ponencia presentada en VI Congreso de la Red latinoamericana de antropología jurídica (Relaju). Diversidad étnica y cultural: desarrollos constitucionales, políticas y prácticas nacionales, Bogotá, 28-31 de octubre, 2008.

Llano Franco, Jairo Vladimir. Teoría del Estado y del derecho. Pluralismo jurídico. Bogotá: Ibáñez, 2017.

López Echeverri, Mayerling. "Privación de la libertad y resocialización en Colombia: ipopulismo punitivo?". Nuevos Paradigmas de las Ciencias Sociales Latinoamericanas 11, núm. 21 (2020): 21-33.

Márquez Restrepo, Martha Lucía. "Colombia, Venezuela y Ecuador: cooperación y coexistencia de cara al posacuerdo" en Política exterior colombiana: escenarios y desafios en el posconflicto, editado por Eduardo Pastrana Buelvas y Hubert Gehrin, 539-566. Bogotá: Pontificia Universidad Javeriana, 2015.

Moncayo Zamudio, Camilo Alberto. "Derechos humanos: ¿De la universalidad a la particularidad?". Nuevos Paradigmas de las Ciencias Sociales Latinoamericanas 10, núm. 19 (enero-junio 2019): 23-38. 
Naranjo Álvarez, Jorge. "La violencia sexual a la luz de la ley de justicia y paz: conceptualización y elementos para su estudio”. Novum Jus 15, núm. 2 (2021): 91-119.

Núñez Ávila, María Dolores. "El reconocimiento de 'la justicia indígena' como agente de la reivindicación de los pueblos indígenas en el marco del pluralismo jurídico". Anuario Iberoamericano de Derecho Internacional Penal 6, núm. 6 (2018): 175-200, DOI: https:// doi.org/10.12804/revistas.urosario.edu.co/anidip/a.7162 (acceso octubre 15, 2021). Oficina Internacional del Trabajo [OIT]. Convenio Núm. 169 de la OIT sobre pueblos indígenas y tribales. Ginebra, 27 de junio de 1989.

Organización de las Naciones Unidas [ONU]. Declaración de las Naciones Unidas sobre los derechos de los campesinos y de otras personas que trabajan en las zonas rurales. Nueva York, 17 de diciembre de 2018.

Organización de las Naciones Unidas [ONU]. Declaración de las Naciones Unidas sobre los derechos de los pueblos indígenas. Nueva York, 13 de septiembre de 2007.

Organización de los Estados Americanos [OEA]. Declaración americana sobre los derechos de los pueblos indígenas. Washington, 14 de junio de 2016.

Organización Internacional del Trabajo [OIT]. "El Convenio 169 de la OIT". https://www. ilo.org/global/docs/WCMS_236378/lang--es/index.htm\#: :text=En\%201989\%2C\%20 despu\%C3\%A9s\%20de\%20dos,Paraguay\%2C\%20Per\%C3\%BA\%20y\%20Venezuela (acceso 20 de mayo, 2021).

Pérez-Salazar, Bernardo. "Construcción de paz en el orden del derecho transnacional penal: el caso colombiano". Utopía y Praxis Latinoamericana 23, núm. 1 (2018): 65-76.

Red Nacional de Agricultura Familiar. "La declaración de los derechos de los campesinos sí podría proteger al campesinado colombiano". https://agriculturafamiliar.co/ladeclaracion-de-derechos-campesinos-si-podria-proteger-al-campesinado-colombiano/ (acceso mayo 25, 2021).

República de Colombia, Corte Constitucional. "Diversidad y pluralismo". https://www. corteconstitucional.gov.co/Transparencia/publicaciones/pluralismo\%202020\%20 v3\%2011220.pdf (acceso abril 30, 2021).

República de Colombia, Corte Constitucional. Auto 651 de 8 de octubre de 2018. Expediente T-6823931. M. P. Gloria Stella Ortiz Delgado.

República de Colombia, Corte Constitucional. Sentencia C-077 de 8 de febrero de 2017. M. P. Luis Ernesto Vargas Silva.

República de Colombia, Corte Constitucional. Sentencia SU-039 de 3 de febrero de 1997. M. P. Antonio Barrera Carbonell.

República de Colombia, Corte Constitucional. Sentencia T-063 de 15 de febrero de 2019. M. P. Antonio José Lizarazo Ocampo.

República de Colombia, Corte Constitucional. Sentencia T-129 de 3 de marzo de 2011. M. P. Jorge Iván Palacio Palacio. 
República de Colombia, Corte Constitucional. Sentencia T-281 de 20 de junio de 2019. M. P. Gloria Stella Ortiz Delgado.

República de Colombia, Corte Constitucional. Sentencia T-414 de 2 de julio de 2015. M. P. Luis Guillermo Guerrero Pérez.

República de Colombia, Corte Constitucional. Sentencia T-436 de 12 de agosto de 2016. M. P. Alberto Rojas Ríos.

República de Colombia, Ministerio del Interior. Resolución 114 de 2019. Bogotá, 27 de noviembre de 2019.

Rodríguez, Gloria Amparo. De la consulta previa al consentimiento libre, previo e informado a pueblos indígenas en Colombia. 2a ed. Bogotá: Universidad del Rosario, 2017.

Sánchez Botero, Esther. "Algunas reflexiones sobre la consulta previa en Colombia" en Consulta previa, principios enfoque metodológico e instrumentalización. Experiencias compartidas y lecciones aprendidas. Bogotá: Red Latinoamericana de Antropología Jurídica (Relaju), 2008.

Sánchez Botero, Esther. "El aporte de la antropología jurídica a la construcción del pluralismo jurídico legal y oficial: ¿ficción persuasiva?" en El peritaje antropológico como prueba judicial, editado por Esther Sánchez Botero y Herinaldy Gómez. Bogotá: Red Latinoamericana de Antropología Jurídica (Relaju), 2018.

Silva García, Germán y Bernardo Pérez-Salazar. "Nuevas estrategias de construcción de la realidad del delito en el orden de las sociedades en red". Utopía y Praxis Latinoamericana 24, núm. 2 extra (2019): 123-132.

Silva García, Germán, Fabiana Irala y Bernardo Pérez-Salazar. "Criminalidad, desviación y divergencia. Una nueva cosmovisión en la criminología del sur”. Revista Latinoamericana de Sociología Jurídica 1, núm. 1 (2020): 8-32.

Silva García, Germán. "Corrupción y derechos humanos. El Estado hacendal y la cleptocracia”. Opción 35, núm. 25 esp. (2019): 12-49. Silva García, Germán, Jairo Vladimir Llano Franco, Nicole Velasco Cano y Angélica Vizcaíno Solano. "El desarrollo de la sociología jurídica latinoamericana”. Opción 35, núm. 25 esp. (2019): 1136-1196.

Silva García, Germán. "Historia de la sociología jurídica latinoamericana. Los que se fueron... y su herencia. Manuel Calvo García". Revista Latinoamericana de Sociología Jurídica 2, núm. 3 (2021): 5-21.

Silva García, Germán. "La administración de justicia: ¿escenario para la protección de los grupos sociales vulnerables?”. Revista Colombiana de Sociología, núm. 26 (2006): 105-123.

Stavenhagen, Rodolfo. Derecho indígena y derechos humanos en América Latina. Ciudad de México: Instituto Interamericano de Derechos Humanos, 2012.

Valero Rico, María Elizabeth. "Reconstrucción del tejido social en Colombia a partir de la niñez víctima del conflicto". Nuevos Paradigmas de las Ciencias Sociales Latinoamericanas 7, núm. 13 (2016): 189-220. 
Velandia Montes, Rafael. "Proceso de paz en Colombia y justicia penal internacional". Revista Latinoamericana de Sociología Jurídica 1, núm. 1 (2020): 346-371.

Velasco Cano, Nicole y Jairo Vladimir Llano Franco. "Derechos fundamentales: un debate desde la argumentación jurídica el garantismo y el comunitarismo", Novum Jus 10, núm. 2 (2016): 35-55.

Vivas-Barrera Tania Giovanna y Bernardo Pérez Salazar. "Sobre la situación de graves violaciones de los derechos humanos y el derecho internacional humanitario en contra de las mujeres en el conflicto armado colombiano" en Derechos humanos, paz y posconflicto en Colombia, editado por Tania Giovanna Vivas-Barrera, 59-104. Bogotá: Universidad Católica de Colombia, 2016.

Vivas-Barrera, Tania Giovanna, Alejandro Quintero Sánchez y Bernardo Pérez Salazar. "Propiedad colectiva de la tierra y movimiento indígena en América Latina". Opción 35, núm. 25 esp. (2019): 1323-1354.

Wolkmer, Antonio Carlos. Pluralismo jurídico. Fundamentos de uma nova cultura no direito. 4a ed. San Paulo: Saraiva, 2015.

Wolkmer, Antonio Carlos. Teoría crítica del derecho desde América Latina. Ciudad de México: Akal, 2017.

Zapata Galvis, Juliana y Jairo Vladimir Llano Franco. "Acuerdo de paz, territorio y diversidad en el norte del Cauca" en Pos-acuerdo y territorio en las comunidades indígenas, afro y campesinas en el norte del Cauca, editado por Jairo Vladimir Llano Franco, Nicole Velasco Cano, Hermán Gómez Gutiérrez y Jenny Marcela Trejos, 43-68, Bogotá: Ibáñez, 2019.De Carvalho, Salo. "Criminología, garantismo y teoría crítica de los derechos humanos: ensayo sobre el ejercicio de los poderes punitivos", Novum Jus 3, núm. 1 (2009): 161-200. 